\title{
Comparison of inherent optical properties as a surrogate for particulate matter concentration in coastal waters
}

\author{
Emmanuel Boss ${ }^{1 *}$, Lisa Taylor ${ }^{1}$, Sherryl Gilbert ${ }^{2}$, Kjell Gundersen $^{3}$, Nathan Hawley $^{4}$, Carol Janzen ${ }^{5}$, Tom Johengen $^{6}$, \\ Heidi Purcell ${ }^{7}$, Charles Robertson ${ }^{8}$, Daniel W. H. Schar ${ }^{9}$, G. Jason Smith ${ }^{10}$, and Mario N. Tamburri1 \\ ${ }^{1}$ School of Marine Sciences, University of Maine, Orono, ME, 04469 \\ ${ }^{2}$ College of Marine Science, University of South Florida, St. Petersburg, FL 33701 \\ ${ }^{3}$ Department of Marine Science, University of Southern Mississippi, Stennis Space Center, MS 39529 \\ ${ }^{4}$ National Oceanic and Atmospheric Administration, Great Lakes Environmental Research Laboratory, Ann Arbor, MI 48108 \\ ${ }^{5}$ Sea-Bird Electronics, Inc., Bellevue, WA 98005 \\ ${ }^{6}$ Cooperative Institute for Limnology and Ecosystems Research, University of Michigan, Ann Arbor, MI 48109 \\ ${ }^{7}$ Department of Naval Architecture and Marine Engineering, University of Michigan, Ann Arbor, MI 48109 \\ ${ }^{8}$ Skidaway Institute of Oceanography, Savannah, GA 31411 \\ ${ }^{9}$ Hawaii Institute of Marine Biology, University of Hawaii, Kaneohe, HI 96744 \\ ${ }^{10}$ Moss Landing Marine Laboratories, Moss Landing, CA 95039 \\ ${ }^{11}$ Chesapeake Biological Laboratory, University of Maryland Center for Environmental Science, Solomons, MD 20688
}

\begin{abstract}
Particulate matter concentration (PM, often referred to as total suspended solids [TSS]) is an important parameter in the evaluation of water quality. Several optical measurements used to provide an estimate of water turbidity have also been used to estimate PM, among them light transmission, backscattering, and side-scattering. Here we analyze such measurements performed by the Alliance for Coastal Technologies (ACT) at various coastal locations to establish whether a given optical method performs better than others for the estimation of PM. All the technologies were found to perform well, predicting PM within less than 55\% relative difference for $95 \%$ of samples $(n=85$, four locations). Backscattering performed best as a predictor of PM, predicting PM with less than $37 \%$ relative difference for $95 \%$ of samples. The correlation coefficient (R) was between 0.96 and 0.98 for all methods with PM data ranging between 1.2 to $82.4 \mathrm{~g} \mathrm{~m}^{-3}$. In addition, co-located measurements of backscattering and attenuation improves PM prediction and provides compositional information about the suspended particles; when their ratio is high, the bulk particulate matter is dominated by inorganic material while when low, dominated by organic material.
\end{abstract}

\section{Introduction}

There is much interest among managers and scientists in quantifying suspended particulate matter concentration (PM,

*Corresponding author: E-mail: emmanuel.boss@maine.edu

\section{Acknowledgments}

Alliance for Coastal Technologies (ACT) is a NOAA-funded program and provided the data for this study. Support for our work on optical properties has been provided by the Environmental Optics and Biology Program, Office of Naval Research (grant N00014-04-1-0235), the University of Maine, the US Geological Survey, and the Weizmann Institute (while on a sabbatical leave). This work has benefited from discussions with lan Walsh, Mike Twardowski, Kevin Ruddick, and Hubert Loisel, and technical assistance from Melissa Bos, Constantine Chigounis, Tim Koles, Travis McKissack, Corey Metcalfe, and Debbie Wells. Comments by three anonymous reviewers have substantially improved this manuscript. This article is Great Lakes Environmental Research Laboratory (GLERL) contribution \# 1535. commonly reported as total suspended solids [TSS]) in aquatic environments. Together with measurements of wave action and current velocity, it is used to determine when sediments and the associated particle bound contaminants (e.g., mercury) are resuspended and transported. In addition, particles affect light propagation and availability to aquatic organisms. Because it is often impractical to measure PM at high temporal and spatial resolution using direct laboratory measurements, surrogates and approaches have been developed to provide such resolution.

Turbidity is a property commonly used to describe water clarity in both marine and freshwater environments, providing a gross assessment of light attenuation due to the presence of suspended material. However, turbidity is often not a direct measure of the quantity of interest, such as suspended sediment, living particles, and nonliving organic matter, but rather a measure of the effect of the suspended particles on the 
optical properties of the water. At present, there are numerous methods for quantifying turbidity (e.g., light attenuation, surface scatter, side scatter, laser diffraction, acoustic back-scatter, Secchi disk, etc.).

Differences in methods of measurement and their individual responses to varying types of suspended material have made the measurement of turbidity difficult to perform in a consistent and standardized way. This has necessitated many public-service agencies (e.g., USGS, EPA, ISO, ASTM, etc.) to define turbidity in very specific terms based on well-defined optical methods of measurement (since optically based approaches have been the most used).

The International Organization for Standardization (ISO) has defined turbidity as "the reduction of transparency of a liquid caused by the presence of undissolved matter" (ISO 1999). Several optical techniques have been approved by the ISO for turbidity measurements, among them measurement of light transmission, side-scattering, and backscattering (ISO 1999). Measurements are performed in the red and infrared regions to minimize contamination by dissolved substances.

Although such standards and definitions were created to be both technically and legally specific (thereby minimizing the ambiguity in interpreting what turbidity is and how it is measured), they still suffer from fundamental deficiencies in their ability to create an absolute standard that covers both different natural water types and different instrument designs. Despite these limitations, a variety of in situ instruments that provide some measure of turbidity are commonly and successfully used in many research and monitoring settings to provide a relative measure of water clarity. However, differences in methods of measurements and their individual response to varying types of $\mathrm{PM}$ have made the measurements of turbidity difficult to standardize. Despite decades of attention to this problem by international and national agencies, the questions of what turbidity is, how it is measured, and what measurements of turbidity tell us have still not yet been resolved (ACT 2005). If turbidity is to be used as a primary variable characterizing water quality, then an evaluation of existing turbidity measurement methods is needed (ACT 2005).

To that end, the Alliance for Coastal Technologies (ACT) sponsored a 3-day workshop with aquatic scientists, managers, and turbidity sensor manufacturers (ACT 2005), establishing protocols for the verification of in situ turbidity sensors (ACT 2006), and conducting a series of laboratory and field deployments of turbidity sensors at sites in the coastal United States.

Many studies have related measurements of a single optical property to PM. Baker and Lavelle (1984), Bishop (1986), and Wells and Kim (1991) showed that for large data sets, the correlation of beam-attenuation and PM are high but that the conversion between the beam-attenuation and PM is constrained; changes in size and composition resulted in poor correlations at specific times and sites. Baker et al. (2001) showed that sidescattering was at least as good a predictor of PM as beam-atten- uation for particles in the deep sea, and that the measurement response to mass was also size dependent. Backscattering has also been shown to be a good predictor of PM while being sensitive to size and composition (Downing 2006 and references therein). Finally, the particulate scattering coefficient at $555 \mathrm{~nm}$ has been found to correlate well with PM for over 200 samples from a variety of European sites; their variable composition was found to have little influence on the relationship (Babin et al. 2003). In addition to composition and size, changes in particle shape (e.g., Clavano et al. 2007), internal structure (e.g., Zaneveld and Kitchen 1995), and degree of packing of aggregates (Boss et al. 2009a) are also expected to affect the relationship between optical properties and PM.

The goal of this paper is to use the database assembled by ACT to compare how different technologies used to measure turbidity in situ (e.g., optical measurements of transmission, side-scattering, and backscattering) perform as proxies of PM. Accuracy and sensitivity of specific instruments and methods are expected to vary due to variation in the measurement pathlength, the size of the volume illuminated, source and detector geometries, and the attenuation of the beam along a path (e.g., Downing 2006). In addition, since these measurements respond differently to the physical (size, shape, internal structure) and optical properties of particles (index of refraction), we demonstrate that using two of these methods together provides information on the bulk particulate composition.

\section{Materials and procedures}

The ACT data set was collected from moored deployments at eight test sites representing a range of environmental conditions including a tropical coral reef, a high turbidity estuary, open ocean, and a freshwater lake. The test sites were situated on a fixed pier (Winans Lake, Ann Arbor, Michigan; Grand Travers Bay, Traverse City, Michigan), a fixed bottom-mounted tripod (a fringing Reef, near Coconut Island within Kaneohe Bay, Hawaii), floating docks (Solomons, Maryland; the Damariscotta River Estuary, Walpole, Maine; Moss Landing Harbor, California; the western shore of Skidaway Island, Georgia), and a piling structure located offshore of Tampa Bay, St. Petersburg, Florida (Fig. 1).

The sensors used in the ACT study consisted of five commercial back- ( $\lambda=700 \mathrm{~nm}$, denoted by $b_{b}$, centered around $\left.120^{\circ}\right)$ and side-scattering $\left(\lambda=880 \mathrm{~nm}\right.$, denoted by $b_{s}$, centered around $90^{\circ}$ ) optical turbidity probes (ACT 2007a-e). A CTD package, an in situ fluorometer, and a beam-transmissometer (Sea-Tech, $\lambda=660 \mathrm{~nm}$, output beam-attenuation denoted by $c$, acceptance angle $1.03^{\circ}$ ) were co-deployed. ACT personnel conducted all tests and analyses in accordance with training provided by the sensor manufacturers and instrument manuals (ACT 2006; ACT 2007a-e).

The turbidity probes were calibrated onsite and deployed side-by-side on a single, box-shaped rack at a fixed depth of 1 $\mathrm{m}$ for continuous time periods ranging from 4-8 weeks. Each sensor recorded data every $15 \mathrm{~min}$. Four of the probes were 


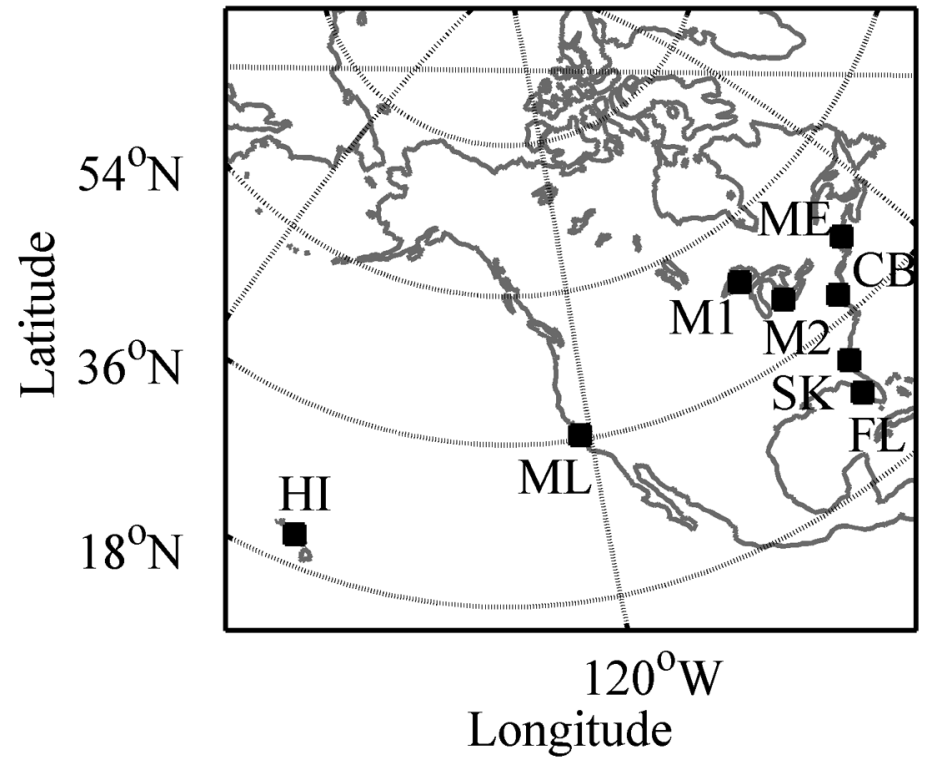

Figure 1. Sample locations. CB: Solomons, Maryland; FL: Tampa Bay, Florida; HI: Kaneohe Bay, Hawaii; M1: Grand Traverse Bay, Lake Michigan; M2: Winans Lake, Michigan; ME: Damariscotta River Estuary, Maine; ML: Moss Landing Harbor, California; SK: Skidaway Island, Georgia.

equipped with integrated wipers to reduce the effects of biofouling, which was estimated qualitatively through the use of photographs taken before deployment and immediately following recovery. Growth substrates (glass and PVC plates) were used to assess biofouling rate. Instrument drift over the whole deployment was evaluated by placing each sensor in a container of turbidity blank (fixed, bubble-free deionized water) and then a second container of 5 NTU suspension of styrene divinylbenzene (SDVB, as reference standards) before and after deployment. For turbidity sensors, calibration involved a linear transfer function converting instrument output to physical units: calibrated-data $=$ (output signal-blank) $\times$ (conversion parameter). The blank was computed at each site using particle-free water and the conversion parameter was computed following the manufacturer's protocol (see ACT $2007 a-e)$. If negative-calibrated data were obtained at a given site with a given instrument, the minimum value measured by this instrument was used as blank for this site. The transmissometer was connected to a data logger and placed in the water near the turbidity probes to collect ancillary data on the particulate beam-attenuation coefficient (c, units are $\left[\mathrm{m}^{-1}\right]$, values reported here do not include the attenuation due to water). Because this instrument is prone to biofouling, it was cleaned five days a week. After cleaning, one in-air value was recorded to assure that the sensor was performing consistently throughout the test period. The data presented here include only data within four hours from cleaning. The transmissometer collected data at the same 15-min intervals as the turbidity probes.

Water samples were collected and analyzed for PM and particulate organic carbon (POC) using a $0.7 \mu \mathrm{m}$ nominal pore size GF/F filter. PM mass was determined gravimetrically for material in accordance with standard protocols (APHA/ AWWA/WPCF 1976 [method 208 D]; EPA 1971; Etcheber 1981) independently at each site including accounting for salt effect (e.g., Stavn et al. 2009). POC samples were measured by the Dumas combustion method at the Marine Science Institute, University of California, Santa Barbara (see ACT 2006 and ACT 2007a-e, for more details).

Data-Only measurements made at the times of the discrete PM sampling were used in the analysis. The number of data points obtained for each of the five turbidity sensors, c, PM, and POC are summarized in Table 1 . Suspect data were removed from the data set before our comparison. These included those measurements collected during sensor failure (characterized by large erratic fluctuation in the signal) or when the sensor experienced obvious drift (low passed signal increases monotonically). Sampling was designed to resolve the dynamic range in PM associated with the tidal dynamics at each environment, as well as longer term changes associated with the weather (e.g., the 5-d mesoscale band), with more intensive sampling earlier in the deployment to provide good reference sample coverage (prior to possible fouling interference).

We selected a subset of the data with the highest number of simultaneous measurements for PM, c, one backscatter sensor, and one side-scatter sensor (95 data points from five locations). Both turbidity sensors used had automated shutters to minimize biofouling. The purpose of pooling the data was to maximize the dynamic range and number of points for the statistical analysis relating specific optical properties and PM. The distribution of the optical properties (OP) for the times where all three sensors and PM data are coincident (95 points) is shown in Fig. 2. As with many environmental variables and optical data (Campbell 1995; Whitmire et al. 2007), the data

Table 1. Number of measurements of PM, POC, $c$ (beam-attenuation), $b_{b}$ (backscatter), and $b_{s}$ (sidescatter), with the number in the subscript denoting sensor type.

\begin{tabular}{|c|c|c|c|c|c|c|c|c|}
\hline Measurements & PM & POC & c & $b_{b 1}$ & $b_{b 2}$ & $\mathbf{b}_{\mathrm{s} 1}$ & $b_{s 2}$ & $\mathbf{b}_{\mathrm{s} 3}$ \\
\hline Collected & 455 & 309 & 251 & 302 & 396 & 409 & 376 & 357 \\
\hline Valid & 455 & 309 & 250 & 248 & 64 & 310 & 221 & 195 \\
\hline Co-located (all methods and sensors) & 21 & 21 & 21 & 21 & 21 & 21 & 21 & 21 \\
\hline Co-located (one sensor of each method) & 95 & 95 & 95 & 95 & & 95 & & \\
\hline
\end{tabular}



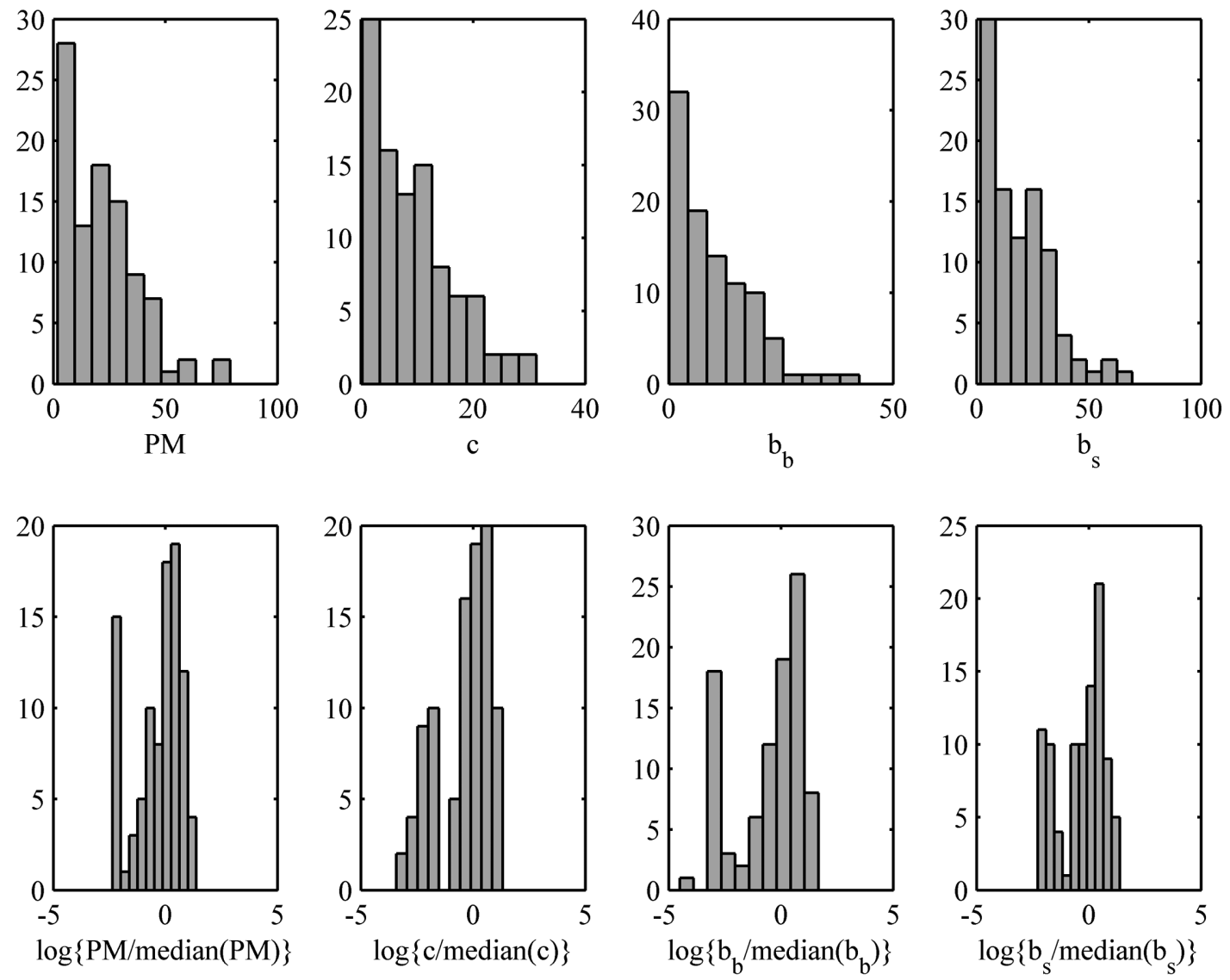

Figure 2. Histograms of frequency distributions of data $(\mathrm{N}=95)$. Values of data within reported units (top panels) and log-normalized data (bottom panels).

variables analyzed are better described by a log-normal distribution than a normal distribution (Fig. 2). Dividing the data by their median and log-transforming them provides nondimensional and normally-distributed variables (e.g., $\log [$ data/median(data) $]$ ).

Since there is no simple statistical formula to calculate the uncertainties in the correlation coefficient between two variables having heterogeneous uncertainties, we used a bootstrap method to obtain the statistics of the correlation coefficient. The original data set and its estimated errors were used to simulate 1000 data sets with the same median. These simulations were then used to derive the statistics of the correlation coefficient (e.g., Press et al. 1992). The correlation coefficients obtained in this manner appear normally distributed, so we used three standard deviations of the correlation coefficients to provide us with the 95\% confidence intervals around the mean.

The uncertainties in the PM are based on the standard deviation of triplicate samples while the uncertainties of the optical measurements for each specific sensor were based on the blank and temperature sensitivities exhibited by the measurements in the predeployment calibrations. For all instruments, these uncertainties are less than seven percent or one turbidity unit, whichever is largest.
The data were collected at sites with variable forcing (weather, tides) and particle sources. At all sites, efforts were made to capture the full extent of the short-term local variability by resolving the tidal variability during the sampling period. Temperature varied from $11.9-31.2^{\circ} \mathrm{C}$ and salinity from $0.2-33$ psu for the data set analyzed here. The sites also varied in their sediment grain size distribution, chlorophyll fluorescence, and POC (ACT 2007a-e).

We used a type-II linear regression analysis (e.g., Laws 1997) between $\log (\mathrm{PM})$ and $\log (\mathrm{OP})$ to devise a model to predict PM from OP for all the instruments that participated in the ACT turbidity study. This analysis is based on York (1966), as implemented in the MATLAB program lsqcubic.m by E. T. Peltzer (for details, see http://www.mbari.org/staff/etp3/regress.htm). The retrieved PM model took into account the uncertainties in the measurements and minimized the squares of the relative difference between PM measurement and model as opposed to the minimization of the square of the difference when linearly regressing OP and PM. For completeness, we also performed this analysis for each sensor individually using all the pairs of PM and optical measurement (see Web Appendix I).

To assess whether measuring two optical properties improved the prediction of PM, we performed a type-I multivariate linear regression analysis between the $\log (\mathrm{PM})$ and the 
logarithm of optical properties, allowing for a constant offset. We used the MATLAB function regress.m and computed the relative error between the multivariate model and measured PM. These models always performed better than standard multivariate linear model in minimizing the relative error between PM and the model.

\section{Assessment}

For the data set combining all sites for which data were available simultaneously with all three technologies, we find correlations between optical properties and PM to be high $(>0.86$, Table 2), with the back- and side-scattering methods being significantly better predictors of PM than beam-attenuation. If the data from Hawaii (which appear optically anomalous, most likely due to high bi-refringent and variable $\mathrm{CaCO}_{3}$ con-
Table 2. Correlation coefficient between data sets $(n=95)$. Uncertainty in the correlation coefficient represents the $95 \%$ confidence interval.

\begin{tabular}{lccc}
\hline $\mathrm{R}(\boldsymbol{n}=\mathbf{9 5}) \pm 3 \mathrm{SD}$ & $\mathrm{c}(\mathbf{6 6 0})$ & $\mathrm{b}_{\mathrm{b}}(\mathbf{7 0 0})$ & $\mathrm{b}_{\mathrm{s}}(\mathbf{8 8 0})$ \\
\hline $\mathrm{PM}$ & $0.86 \pm 0.09$ & $0.92 \pm 0.11$ & $0.93 \pm 0.06$ \\
\hline
\end{tabular}

tent, Fig. 3) are removed, we find the correlations to be significantly higher for all variables $(>0.96$, Table 3$)$ with backscattering having a slightly better correlation over side-scattering and beam-attenuation.

For the type-II model based on backscattering data, we find that $50 \%$ of the model-predicted PMs are within $9 \%$ of measured PM and 95\% of the model-predicted PMs are
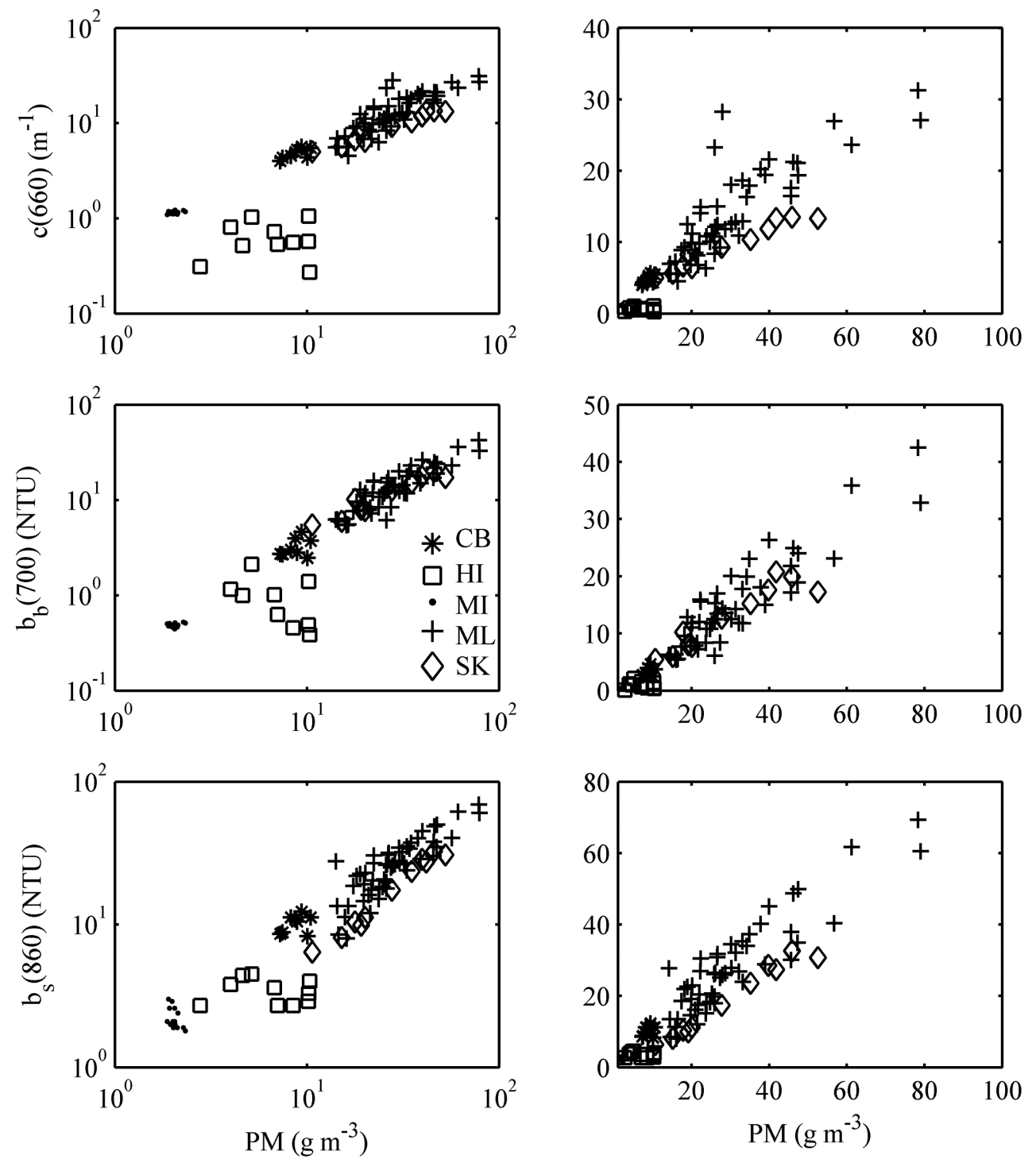

Figure 3. Value of optical measurements (in units of $m^{-1}$ for $c$ and NTU for $b_{b}$ and $b_{s}$ ) as function of PM including all collocated measurements $(N=$ 95). Symbol denotes sampling site. The same data is presented in both a log-log plot (left panels) and a linear plot (right panels). 
Table 3. Correlation of data sets without Hawaii $(n=85)$. Uncertainty in the correlation coefficient represents the $95 \%$ confidence interval.

\begin{tabular}{lccc}
\hline$R(n=85) \pm 3$ SD & $c(660)$ & $b_{b}(700)$ & $b_{s}(880)$ \\
\hline$P M$ & $0.970 \pm 0.005$ & $0.982 \pm 0.005$ & $0.962 \pm 0.008$
\end{tabular}

Table 4. Statistics of the ratio of the absolute value of the difference between PM from a type-II regression model based on an optical property and the measured PM divided by the measured PM (the prediction percentile error) for the same data as in Table 3. Values on the left-most column are the percent of observation. The value at the intersection of a population percentile row and the optical property column denote the prediction percentile error associated with the two, e.g., for $95 \%$ of the observation, a PM model based on backscattering measurements agrees with the observed PM within 36\%.

\begin{tabular}{lccc}
\hline $\begin{array}{l}\text { Prediction percentile error, } \\
\text { IModel-PMI/PM }\end{array}$ & $\mathbf{c ( 6 6 0 )}$ & $\mathbf{b}_{\mathbf{b}} \mathbf{( 7 0 0 )}$ & $\mathbf{b}_{\mathbf{s}}(\mathbf{8 8 0})$ \\
\hline $\begin{array}{l}\text { Population percentiles } \\
5 \%\end{array}$ & $2 \%$ & $1 \%$ & \\
$50 \%$ & $16 \%$ & $9 \%$ & $21 \%$ \\
$95 \%$ & $54 \%$ & $36 \%$ & $51 \%$ \\
\hline
\end{tabular}

within $36 \%$ of measured PM (Table 4). The agreement is not as good for either the beam-attenuation or the side-scattering measurements (Table 4). These results have been obtained for waters varying nearly two orders of magnitude in PM concentration (1.2-80 $\left.\mathrm{mg} \mathrm{L}^{-1}\right)$. Type-I multivariate models including two optical properties perform slightly better than backscattering alone (predicting PM with an error less than $35 \%$ for $95 \%$ of the data), and only if the backscattering coefficient is one of the two optical properties measured (Table 5).

The above analysis is quasi-independent of calibration constants applied (quasi-independent due to log-transforming a linear function of the measured signal). It assumes, however, that no change in the calibration coefficients of the instruments occurred within each deployment (e.g., we assume instrumental stability) and that instrument linearity was maintained throughout the range of measurements (at very high concentrations, attenuation of the beam along the photon path from source to detector can result in a decrease of signal with concentration for scattering sensors).

\section{Discussion}

The primary goal of this paper is to compare how different optical technologies used to measure turbidity in situ perform as surrogates of PM. Similar previous investigations were limited to comparing measurements made with a single method over a large range of conditions (e.g., Baker and Lavelle 1984; Babin et al. 2003; Downing 2006).
Table 5. Statistics of the ratio of the absolute value of the difference between PM from a type-I multivariate regression model based on two optical properties and the measured PM divided by the measured PM (the prediction percentile error) for the same data as in Table 3. Values on the left-most column are the percent of observation. The value at the intersection of a population percentile row and the optical properties column denote the prediction percentile error associated with the two, e.g., for $95 \%$ of the observation, a PM model based on backscattering, and sidescattering measurements agree with the observed PM within 35\%.

\begin{tabular}{|c|c|c|c|}
\hline $\begin{array}{l}\text { Prediction percentile error, } \\
\text { IModel-PMI/PM }\end{array}$ & $\begin{array}{c}c(660) \\
\text { and } \\
b_{b}(700)\end{array}$ & $\begin{array}{c}c(660) \\
\text { and } \\
b_{s}(880)\end{array}$ & $\begin{array}{c}b_{s}(880) \\
\text { and } \\
b_{b}(700)\end{array}$ \\
\hline \multicolumn{4}{|l|}{ Population percentiles } \\
\hline $5 \%$ & $0.5 \%$ & $2 \%$ & $1 \%$ \\
\hline $50 \%$ & $10 \%$ & $17 \%$ & $11 \%$ \\
\hline $95 \%$ & $36 \%$ & $42 \%$ & $35 \%$ \\
\hline
\end{tabular}

We observe that while differences between the ability of optical properties to predict PM are small, they are significant (Table 3); the backscattering coefficient outperforms the beam-attenuation and side scattering in the prediction of PM. We currently, however, do not have a definitive explanation for this performance difference. Hypotheses include possible larger effect of dissolved substances on beam-attenuation than scattering measurements (due to longer pathlength and shorter wavelength) or size effects on scattering within the acceptance angle of the beam transmissometer resulting in large particles contributing less per mass compared to backscattering (Boss et al. 2009b). We cannot rule out, however, that differences in handling (manual cleaning of the transmissometer vs. automated shutter for the turbidity sensors) could explain the observed differences. An automated method to minimize biofouling of flow-through transmissometers does exist (Manov et al. 2004) but cannot be applied to transmissometers open to the environment as was done here. Measuring two optical properties improves retrieval of PM, though not by much, and only if backscattering is one of the two optical properties measured (Table 5).

In general, however, measuring a single optical property provides a good prediction of PM (e.g., the uncertainty is less than $55 \%$ for $95 \%$ of the cases with all optical methods) for our data set. A question arises whether measuring with more than one optical property provides additional information regarding the particles. Since, it was observed in past work (Twardowski et al. 2001; Boss et al. 2004; Sullivan et al. 2005; Loisel et al. 2007) that the ratio of particulate backscattering to total scattering provides information regarding the composition of particles we attempted to correlate the ratio of backscattering and beam attenuation to the ratio of POC/PM. We found the two ratios to inversely vary, as predicted by theory (Fig. 4, and see Twardowski et al. 2001; Boss et al. 2004) and observed by Loisel et al (2007) in the English Channel (his 


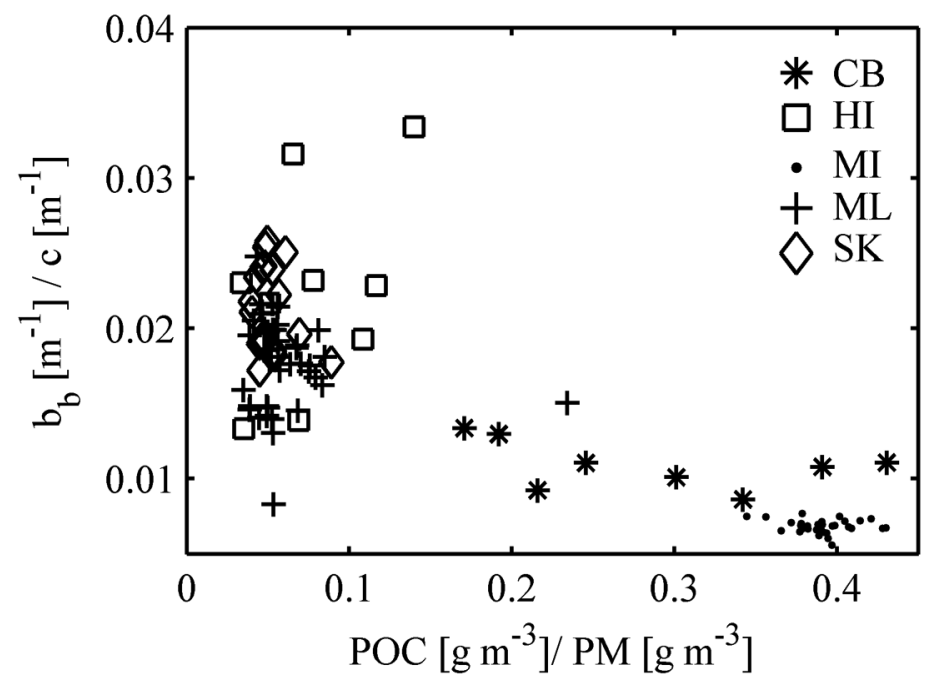

Figure 4. Ratio of $b_{b}(700)$ to $c(660)$ as function of the ratio of POC to PM including all collocated measurements of $b_{b}$ and $c(N=128)$. A factor of $0.0163\left[\mathrm{~m}^{-1}\right] /$ NTU was used to convert the $b_{b}(700)$ measurement units, to facilitate comparison with published values of $b_{b} / c$.

Table 2) for the ratio of the backscattering to the total scattering coefficient.

\section{Comments and recommendations}

Analysis of the study data set suggests that all the optical methods used as a surrogate of PM perform well (relative error smaller than $55 \%$ for $95 \%$ of the cases in widely varying environments). Backscattering is the method providing the best prediction of PM in this data set (relative error smaller than $37 \%$ for $95 \%$ of the cases).

We recommend (as did others, e.g. Gibbs 1974; Zaneveld et al. 1979; Downing 2006) that the use of turbidity standards for assessment of PM be stopped and that efforts be focused on calibrating with PM, a biogeochemical variable of direct link to water quality. Different instruments calibrated with the same turbidity standard in the lab may not give the same results in the field and the differences may be very large (Gibbs 1974; Downing 2006). Standards could continue to be used to monitor instrument drift, and, if their optical properties are known, to calibrate sensors to absolute physical units (which could then be used for comparison with theory).

The need for calibration stems from the difference between sensor designs, even when they measure a similar optical property. For example, we recently published an intercomparison of commercial transmissometers. Changes in acceptance angle between two sensors of the same manufacturer caused an $18 \%$ change in the estimated beam-attenuation for openocean measurements. In coastal waters, the differences were significantly larger and tide phase dependent (Boss et al. 2009b). Similar differences exist between backscattering sensors (Downing 2006). Thus, the correlation of an optical mea- surement with PM is most often sensor-specific. In the appendix, we provide the regressions for the specific sensors that participated in the ACT Technology Evaluation using all the matchup data that were available for that specific sensor (size and locations of data set varied between sensors).

If possible, we also recommend the use of several concurrent optical methods to estimate PM. Besides the advantage of redundancy in case of malfunction and improvement in PM prediction (Table 5), the additional data can provide added information about the particles such as composition and/or size distribution.

\section{References}

ACT [Alliance for Coastal Technologies]. 2005. Measuring turbidity in coastal waters. ACT WR05-08, UMCES/CBL 06-026.

- 2006. Protocols for verifying the performance of in situ turbidity sensors, ACT PV06-01, UMCES/CBL 07-048.

- 2007a. Performance verification statement for the aquatec turbidity sensor. ACT TV07-01, UMCES/CBL 07-049. - 2007b. Performance verification statement for the insitu turbidity sensor. ACT TV07-02, UMCES/CBL 07-050.

. 2007c. Performance verification statement for the mcvan turbidity sensor. ACT TV07-03, UMCES/CBL 07-051. . 2007d. Performance verification statement for the wet labs turbidity sensor. ACT TV07-04, UMCES/CBL 07-052.

- 2007e. Performance verification statement for the YSI turbidity sensor. ACT TV07-05, UMCES/CBL 07-053.

[APHA/AWWA/WPCF] American Public Health Association, American Water Works Association, Water Pollution Control Federation. 1976. Standard methods for the examination of water and wastewater, 14th ed. APHA, AWWA, and WPCF. Method 208 D. Total nonfilterable residue dried at 103-105 C (total suspended matter).

Babin, M., A. Morel, V. Fournier-Sicre, F. Fell, and D. Stramski. 2003. Light scattering properties of marine particles in coastal and oceanic waters as related to the particle mass concentration. Limnol. Oceanogr. 48:843-859.

Baker, E. T., and J. W. Lavelle. 1984. The effect of particle size on the light attenuation coefficient of natural suspensions. J. Geophys. Res. 89:8197-8203.

Baker, E. D., A. Tenant, R. A. Feely, G. T. Lebon, and S. L. Waker. 2001. Field and laboratory studies on the effect of particle size and composition on optical backscattering measurements in hydrothermal plumes. Deep Sea Res. I 48:593-604.

Bishop, J. K. B. 1986. The correction and suspended particulate matter calibration of Sea Tech transmissometer data. Deep Sea Res. 33:121-134.

Boss, E., W. S. Pegau, M. Lee, M. S. Twardowski, E. Shybanov, G. Korotaev, and F. Baratange. 2004. Particulate backscattering ratio at LEO 15 and its use to study particles composition and distribution. J. Geophys. Res. 109:C01014 [doi:10.1029/2002JC001514].

— W. H. Slade, and P. Hill. 2009a. Effect of particulate aggre- 
gation in aquatic environments on the beam-attenuation and its utility as a proxy for particulate mass. Opt, Expr. 17:94089420.

, - M. Behrenfeld, and G. Dall'Olmo. 2009b. Acceptance angle effects on the beam-attenuation in the ocean. Opt. Expr. 17:1535-1550.

Campbell, J. W. 1995. The lognormal distribution as a model for bio-optical variability in the sea. J. Geophys. Res. 100:13237-13254.

Clavano, W. R., E. Boss, and L. Karp-Boss. 2007. inherent optical properties of non-spherical marine-like particles - from theory to observations. Oceanogr. Mar. Bio. Ann. Rev. 45:1-38.

Downing, J. 2006. Twenty-five years with OBS sensors: The good, the bad, and the ugly. Cont. Shelf Res. 26:2299-2318.

[EPA] U.S. Environmental Protection Agency. (1971). U.S.EPA National Exposure Research Laboratory (NERL) method 160.2. Residue, non-filterable (gravimetric, dried at 103-105). <http://www.nemi.gov/apex/f?p=237:38:185126857084961:::: P38_METHOD_ID:5213>.

Etcheber, H. 1981. Comparaison de diverses methods d'évaluation des teneurs en matières en suspension et en carbone organique particulaire des eaux marines du Plateau Continental Aquitain. J. Rech. Oceanogr. 6:37-42.

Gibbs, R. J. 1974. Principles of studying suspended materials in water, p. 3-15. In R. J. Gibbs [ed.], Suspended solids in water. Plenum.

[ISO] International Organization for Standardization. 1999. Water quality — Determination of turbidity. ISO 7027:1999(E). ISO.

Laws, E. 1997. Mathematical methods for oceanographers. Wiley.

Loisel, H., X. Mériaux, J. F. Berthon, and A. Poteau. 2007. Investigation of the optical backscattering to scattering ratio of marine particles in relation with their biogeochemical composition in the eastern English Channel and southern North Sea. Limnol. Oceanogr. 52:739-752.

Manov, D.V., G.C. Chang, and T.D. Dickey. 2004. Methods for reducing biofouling of moored optical sensors. J. Atmos. Oceanic Tech. 21:957-967.

Press, W. H., A. Teukolsky, W. T. Vetterling, and B. P. Flannery. 1992: Numerical recipes in C. Cambridge.

Stavn, R. H., H. J. Rick, and A. V. Falster. 2009. Correcting the errors from variable sea salt retention and water of hydration in loss on ignition analysis: Implications for studies of estuarine and coastal waters. Est. Coast. Shelf Sci. 81:575-582.

Sullivan, J. M., M. S. Twardowski, P. L. Donaghay, and S. A. Freeman. 2005. Use of optical scattering to discriminate particle types in coastal waters. Appl. Opt. 44:1667-1680.

Twardowski, M., E. Boss, J. B. MacDonald, W. S. Pegau, A. H. Barnard, and J. R. V. Zaneveld, 2001. A model for estimating bulk refractive index from the optical backscattering ratio and the implications for understanding particle composition in case I and case II waters. J. Geophys. Res. 106:14129-14142.

Wells, J. T., and S. Kim. 1991. The relationship between beam transmission and concentration of suspended particulate material in the Neuse River Estuary, North Carolina. Estuaries 14:395-403.

Whitmire, A. L., E. Boss, T. J. Cowles, and W. S. Pegau. 2007. Spectral variability of the particulate backscattering ratio. Opt. Expr. 15:7019-7031.

York, D. 1966. Least-squares fitting of a straight line. Can. J. Phys. 44:1079-1086.

Zaneveld, J. R. V., R. W. Spinrad, and R. Bartz. 1979. Optical properties of turbidity standards. Proc. Soc. Photo. Opt. Instrum. Eng. 208(6):159-168.

, and J. C. Kitchen. 1995. The variation in the inherent optical properties of phytoplankton near an absorption peak as determined by various models of cell structure. J. Geophys. Res. 100:13309-13320.

Submitted 22 October 2008 Revised 28 September 2009 Accepted 7 October 2009 